\title{
Erratum: Sharing of nonlocal advantage of quantum coherence by sequential observers [Phys. Rev. A 98, 042311 (2018)]
}

\author{
Shounak Datta and A. S. Majumdar
}

(Received 27 December 2018; published 16 January 2019)

DOI: 10.1103/PhysRevA.99.019902

We correct an error in the original paper in determining the conditional state on Bob's side after the sequential measurements performed on the other side. Considering the initial state between Alice ${ }^{1}$ and Bob $\sigma_{A_{1} B}$ after performing a particular unsharp measurement from $\left\{E_{i}^{ \pm}\right\}(i \in\{x, y, z\})$ by Alice ${ }^{1}$, the conditional state on Bob's side becomes

$$
\sigma_{B \mid E_{i}^{ \pm}}=\frac{\operatorname{Tr}_{A}\left[\left(\sqrt{E_{i}^{ \pm}} \otimes \mathbb{1}_{2}\right) \sigma_{A_{1} B}\left(\sqrt{E_{i}^{ \pm}} \otimes \mathbb{1}_{2}\right)\right]}{\operatorname{Tr}\left[\left(\sqrt{E_{i}^{ \pm}} \otimes \mathbb{1}_{2}\right) \sigma_{A_{1} B}\left(\sqrt{E_{i}^{ \pm}} \otimes \mathbb{1}_{2}\right)\right]},
$$

where $E_{i}^{ \pm}=\lambda P_{i}^{ \pm}+\frac{1-\lambda}{2} \mathbb{1}_{2}$ and $E_{i}^{+}+E_{i}^{-}=\mathbb{1}_{2}$. State $\sigma_{B \mid E_{i}^{ \pm}}$occurs with probability $p\left(\sigma_{B \mid E_{i}^{ \pm}}\right)=\operatorname{Tr}\left[\left(E_{i}^{ \pm} \otimes \mathbb{1}_{2}\right) \sigma_{A_{1} B}\right]$. Using the above conditional state on Bob's side, Eq. (15) becomes

$$
N_{A_{1} B}^{l_{1}}=3 \lambda_{1},
$$

which gives a violation of Eq. (5) when $\lambda_{1}>\sqrt{\frac{2}{3}} \simeq 0.82$. By applying a similar argument for Alice ${ }^{2}$, Eq. (17) becomes

$$
\overline{N_{A_{2} B}^{l_{1}}}=\lambda_{2}\left(1+2 \sqrt{1-\lambda_{1}^{2}}\right) \text {. }
$$

So, if $\lambda_{2}=1, \overline{N_{A_{2} B}^{l_{1}}}>\sqrt{6}$ when $\lambda_{1}<0.69$. Hence, no region for $\lambda_{1}$ within $[0,1]$ can be found where both Alice ${ }^{1}$ and Alice ${ }^{2}$ can achieve nonlocal advantage of quantum coherence (NAQC) with Bob using the $l_{1}$ norm. Or in other words, at most, one Alice can exhibit NAQC with Bob. Equations (18) and (19) are thereby rendered irrelevant.

Similarly, for relative entropy of NAQC, the right-hand side of Eq. (20) will be changed to

$$
N_{A_{1} B}^{E}=3 \log _{2}(e) \lambda_{1} \tanh ^{-1}\left(\lambda_{1}\right)+\frac{3}{2} \log _{2}\left(1-\lambda_{1}^{2}\right),
$$

which violates Eq. (7) when $\lambda_{1}>0.91$. Now following Eq. (21), the right-hand side of Eq. (22) becomes

$$
\begin{aligned}
\overline{N_{A_{2} B}^{E}}= & \log _{2}(e) \lambda_{2} \tanh ^{-1}\left(\lambda_{2}\right)+\frac{1}{2} \log _{2}\left(1-\lambda_{2}^{2}\right)+2 \log _{2}(e) \sqrt{1-\lambda_{1}^{2}} \lambda_{2} \\
& \times \tanh ^{-1}\left(\sqrt{1-\lambda_{1}^{2}} \lambda_{2}\right)+\log _{2}\left[1-\left(1-\lambda_{1}^{2}\right) \lambda_{2}^{2}\right],
\end{aligned}
$$

which, in the range of $\lambda_{1} \in(0.91,1]$, gives the quantum mechanical (QM) maximum 1.24 . Therefore, the inference that only one Alice can share relative entropy of NAQC with a single Bob remains unaffected.

Similarly, again, for skew information of NAQC, Eq. (23) turns out to be

$$
N_{A_{1} B}^{S}=3\left(1-\sqrt{1-\lambda_{1}^{2}}\right),
$$

which violates Eq. (9) when $\lambda_{1}>\frac{2 \sqrt{2}}{3} \simeq 0.94$. Using Eq. (24), now Eq. (25) becomes

$$
\overline{N_{A_{2} B}^{S}}=3-\sqrt{1-\lambda_{2}^{2}}-2 \sqrt{1-\left(1-\lambda_{1}^{2}\right) \lambda_{2}^{2}} \text {. }
$$

In the range of $\lambda_{1}>0.94$, the QM maximum of $\overline{N_{A_{2} B}^{S}}$ is 1.11 . Here also the conclusion that at most one Alice can exhibit skew information of NAQC with a single Bob remains unaltered.

Typographical errors in Eq. (16) are corrected by replacing $E_{j}^{+}$by $P_{j}^{+}$and $E_{j}^{-}$by $P_{j}^{-}$.

To conclude, we now get uniform results using all the three measures of coherence, i.e., at most one Alice can reveal NAQC with Bob on the other side. This fits well with the conjecture that, as the strength of quantum correlations increases, the bound 
on the number of observers exhibiting such correlations decreases, viz. from three using a three-settings steering inequality [1] to two for Bell-Clauser-Horne-Shimony-Holt violation [2] and one for NAQC in our paper.

We thank X. Wang, NJUPT (China) for discussions enabling us to fix the error in our calculations.

[1] S. Sasmal, D. Das, S. Mal, and A. S. Majumdar, Phys. Rev. A 98, 012305 (2018).

[2] S. Mal, A. S. Majumdar, and D. Home, Mathematics 4, 48 (2016). 